\title{
Long-term survival after perforated diverticulitis
}

J Vermeulen

MP Gosselink

WCJ Hop

E van der Harst

BE Hansen

GHH Mannaerts

PPLO Coene

WF Weidema

JF Lange

All correspondence to:

Jefrey Vermeulen MD PhD

Erasmus University Medical Centre

Department of Trauma Surgery

Dr Molewaterplein 50

3015 GE Rotterdam

The Netherlands

Email: j.vermeulen.1@erasmusmc.nl

Presented at:

Autumn meeting of the Dutch Society of Surgery 2008, Reehorst (28 nov 2008)

\section{Abstract}

Short-term survival after emergency surgery for perforated diverticulitis is poor. Less is 
known about long-term survival. The aims of this study were to evaluate long-term survival after discharge from hospital and to identify factors associated with prognosis.

All patients who underwent emergency surgery for perforated diverticulitis in five hospitals of Rotterdam, The Netherlands between 1990 and 2005 were included. The association between type of surgery (Hartmann's procedure or primary anastomosis) and long-term survival was analysed by multivariate Cox's regression analysis taking account of age, American Society of Anesthesiology (ASA) classification, Hinchey score, Mannheim Peritonitis Index (MPI) and surgeon's experience. In addition, survival of the patients was compared to the matched general Dutch population.

A total of 340 patients were identified. Of these patients $49 \%$ were classified ASA III or IV. The overall 5-years survival was 53\%. 250 patients were discharged from hospital alive after primary surgery. Median time of follow up of these 250 patients was 59 months. During follow up another 90 patients (58\% was older than 75 year; 66\% ASA III/IV) died. Survival was significantly impaired compared to the expected matched sex-, age- and calendar timespecific survival. Overall survival was significantly related to age and ASA classification. Hinchey score, MPI, number of re-interventions, the surgeon's experience and type of surgery did not influence long-term survival, although a trend was found towards Hartmann's procedure being a risk factor for poorer survival compared to primary anastomosis (hazard ratio for mortality $1.88 ; 95 \% \mathrm{CI} 0.96-3.67 ; \mathrm{p}=0.07$ ).

Long-term survival of patients after perforated diverticulitis is limited and mainly caused by the poor general condition of the patients and not by the severity of primary disease or year and type of surgery.

\section{Introduction}

Diverticular disease of the colon is common in westernized countries, especially in older 
people [1,2]. The large majority of patients remain asymptomatic. Only 1-2\% of patients presenting for urgent abdominal evaluation have free perforation because of diverticulitis [3]. Nevertheless, the prevalence of perforated diverticulitis is increasing [4], which parallels the ageing of the developed countries.

Emergency operations for perforated diverticulitis are associated with substantial mortality (826\%) [5-8], which can increase up to $40 \%$ in the elderly [8,9]. The rate of severe postoperative complications is also high, which reflects the severity of the disease and the poor general condition of the patients [10]. After surviving primary surgery patients commonly face a long period of rehabilitation. Probably the majority of patients will never return to their prehospital health state because of postoperative side effects and complications. This might result into a shorter life expectancy.

The existing literature regarding perforated diverticulitis only reports about short-term postoperative mortality and postoperative complications. Direct postoperative mortality is known to be influenced by age and American Society of Anesthesiology (ASA) classification of the patient, the severity of the disease indicated by Hinchey score and Mannheim Peritonitis Index (MPI), type of surgery and surgeon's experience in colorectal surgery [7-10]. Less is known about survival outside the hospital on the long term. Survival expectancy could be an important factor in decision making for initial (surgical) treatment as it measures the patient's prognosis. The present study was undertaken to determine the long-term survival of patients who were operated for acute perforated diverticulitis. The issues of surgical technique, severity of primary disease and patients' characteristics will be addressed and compared with natural life span of the Dutch population.

\section{Patients and methods}

A cohort of 340 consecutive patients were included provided they had undergone emergency surgery for perforated diverticulitis between January 1990 and December 2005 at the surgical departments of the academic and the four major teaching hospitals of Rotterdam, The Netherlands. Patients admitted for perforated diverticulitis could be identified and selected from computerized surgery registration databases. The indications for surgery were clinical signs of diffuse peritonitis or the presence of sepsis with acute abdominal pain, free gas on plain abdominal radiography or specific findings at ultrasonography or computerized 
tomography. The type of surgical procedure was left to the discretion of the surgeon on call. As the operations were classified as emergency and hence frequently performed outside office hours, not all procedures were performed by specialist colorectal surgeons. Surgery consisted of Hartmann's procedure (HP; $n=238$ ), resection with primary anastomosis (PA; $n=93$ ), suture repair $(n=7)$ and the performance of a diverting stoma without resection $(n=2)$.

The database included patients' characteristics, year and type of surgical procedure, surgeon's experience, MPI, Hinchey score, postoperative medical and surgical adverse events, number of re-interventions, length of hospitalization and intensive care unit stay, the date of eventual death and the cause of death. Severity of disease was stratified with MPI [11] and Hinchey scores [12]. MPI was calculated from the medical reports of all patients, whereas Hinchey score was distracted from the surgical reports. A MPI=26 is known to be a significant predictor of mortality [11]. In this study, all additional radiological interventions or reoperations after primary emergency surgery were recorded as re-interventions. These were defined as radiological-assisted percutaneous drainage of abdominal or pelvic abscess, open abdominal wound management or re-operations owing to ongoing sepsis, abdominal abscess formation, abdominal dehiscence, anastomotic leakage or stoma-related complications.

Follow up of all patients was conducted to July 2007. Data about the patients' course after initial hospital discharge were gathered from the medical reports and by telephone enquiry performed from the patients themselves, or when necessary, the patient's direct relatives or general practitioner. Patients lost to follow up were censored at the time of their last hospital visit. Long-term survival of patients who were operated for acute perforated diverticulitis was evaluated in relation to patient's characteristics, severity of the disease, type of surgery and postoperative course.

\section{Statistical analysis}

Kaplan-Meier survival plots with log-rank statistics were used in order to determine survival rates univariately according to various factors. The association between type of surgery (HP or PA) and long-term survival was analysed using multivariate Cox's regression analysis taking account of age, gender, ASA classification, Hinchey score and MPI. These analyses were performed with Statistical Package for Social Sciences version 15.0 (SPSS Inc., Chicago, Illinois, USA, 1998). In addition, observed survival was compared with expected survival from matched sex-, age- and calendar time-specific death rates for the general Dutch population using life table method and Wilcoxon (Gehan) test. A test for a single proportion with continuity correction was used to evaluate differences between observed survival and 
survival predicted by the Mayo model (Mayo risk score) [13]. Differences were considered significant at a two-sided $\mathrm{p}$-value $<0.05$.

\section{Results}

Between January 1990 and December 2005, 340 patients underwent emergency surgery for perforated diverticulitis. Of these 90 (26\%) (HP: $n=75$; PA: $n=13$; other: $n=2)$ died during their postoperative period at initial hospital stay. Characteristics of the 250 survivors are shown in table 1. At a median follow up of 59 months (range $1-210$ ) after hospital discharge another 90 patients (36\%; HP: $n=68$; PA: $n=18$; other: $n=4$ ) died. Causes of death are shown in table 2. Survival of all initial 340 patients is shown in figure 1. The overall 5-years survival of the total group ( $\mathrm{n}=340)$ was $53 \%$.

Of the 340 initial patients $49 \%$ was classified ASA III or higher, 33\% was older than 75 years of age and 58\% had generalized peritonitis because of perforation (Hinchey III 42\%; Hinchey IV 16\%). In these patients direct postoperative mortality was very high (41, 34 and 31\%, respectively). In-hospital mortality of patients with Hinchey IV perforated diverticulitis was $48 \%$ (26/54). Long-term follow up of the survivors $(n=250)$ revealed a mortality of $66 \%$ in ASA III or higher and $58 \%$ in patients older than 75 years. Long-term survival of patients who survived initial generalized peritonitis (Hinchey III and IV) was $41 \%$, which is comparable to the whole survivors group $(n=250)$.

Univariate and multivariate analysis of factors, which were considered potentially important in predicting survival of the 250 patients who survived beyond the perioperative period, were performed. In univariate analysis, age and ASA classification (figure 2 and 3) were both significantly associated with long-term survival (both $\mathrm{p}<0.001$ ). Gender was not significantly related to long-term survival after perforated diverticulitis $(\mathrm{p}=0.91)$.

No relation was observed between the severity of the primary disease at initial surgery and prognosis outside the hospital. Hinchey score had no influence on long-term survival after hospital discharge ( $\mathrm{p}=0.27$; figure 4$)$. Also, discharged patients who had MPI $=26$ at initial surgery did not have a worse prognosis on the long term as compared with patients with MPI $<26(p=0.31)$. The need for re-interventions or re-operations to treat postoperative surgical complications after primary surgery during initial hospital stay was not related to long-term survival $(\mathrm{p}=0.53)$ 
Type of initial surgery for perforated diverticulitis was related with long-term survival. Patients who underwent PA had better prognosis compared with those who underwent HP ( $\mathrm{p}=0.005$; figure 5). However, patients who underwent HP generally had a higher age and ASA classifications and had higher Hinchey scores and MPI at primary surgery (all $p<0.001$ ) in comparison with patients who underwent PA. When adjusting for these factors using multivariate analysis, long-term survival rates of PA and HP were not significantly different from each other. A trend was found towards patients who underwent HP being associated with poorer survival ( $\mathrm{p}=0.07$; table 3 ). Similar results were observed when Hinchey score was replaced by MPI in the multivariate analysis. The time period in which the operation had taken place was not related to long-term survival ( $\mathrm{p}=0.27$; figure 6 ), nor was the experience of the operating surgeon and $(\mathrm{p}=0.79)$.

Long-term survival of the in-hospital survivors after perforated diverticulitis was significantly impaired compared to the expected matched sex-, age- and calendar time-specific survival of the general Dutch population ( $\mathrm{p}=0.005$; figure 7$)$. This poorer overall survival seemed to be due to the poor survival of patients after HP ( $\mathrm{p}=0.02$; figure 8$)$. Survival of patients who had undergone PA was not significantly different as compared to the sex-, age- and calendar timematched control group of the general Dutch population ( $\mathrm{p}=0.85$; figure 9 ).

\section{Discussion}

The present study demonstrates reduced long-term survival for patients who survived initial emergency surgery for treatment of perforated diverticulitis compared with the general population. Besides a high direct-postoperative mortality rate $(26 \%)$, each year about $5 \%$ of the patients died after having survived initial surgery and discharge from hospital. The observed 5-years survival after perforated diverticulitis was only 53\%. The main reason for this observation is the poor general condition of the patients. Almost half of the patients that presented with acute perforated diverticulitis was classified ASA III or IV and one third was older than 75 years of age. The incidence of perforated diverticulitis is highest in older patients who suffering from multiple comorbidities as indicated by the higher ASA classifications [14]. These factors are not mutable and are known factors for mortality. Mortality after emergency surgery for perforated diverticulitis can increase to $40 \%$ in older patients $[8,9]$. It is therefore not surprising that these have a poor prognosis in the short and 
long term.

Besides patient-related risk factors, also disease related risk factors like Hinchey score and MPI are also known independent risk factors for in-hospital mortality after emergency surgery [15]. The overall in-hospital mortality rate of patients with generalized peritonitis because of perforation was over $30 \%$ and increased towards almost $50 \%$ in cases with faecal peritonitis (Hinchey IV). However, when the patient survived primary surgery and was discharged from the hospital, the severity of disease at primary surgery did not appear to effect long-term survival. The need for one or more radiological-assisted drainage of abdominal abscesses or re-operations for ongoing sepsis, anastomotic leakage or stoma-related complications also did not influence long-term survival after hospital discharge. It seems that in surviving patients, initial severity of disease and the need for additional interventions do not affect the long-term outcome. Surgical experience and the year in which the operation took place were also not related with long-term survival.

The same was observed for the type of surgical procedure. In contrast with univariate analysis in which HP was related with poorer outcome in the long term compared with PA, multivariate analysis, adjusting for the differences in patients' characteristics, severity of disease and surgeon's experience, showed that type of surgery was no longer significantly related to long-term survival. Nevertheless a trend was found for better survival after PA. Compared with the general population survival after HP was restricted, whereas survival after PA was not. The more severely affected and high-risk patients are inclined to undergo HP $[10,16]$. In this study significant higher disease-severity scores (e.g. Hinchey score and MPI), older age and higher ASA classifications were observed in the patients who underwent HP compared with the patients with PA [15]. This could explain the poorer survival of the patients after HP and the similar survival of patients after PA compared with the general population.

As the optimal surgical strategy to treat perforated diverticulitis is still a matter of debate, it is important to be informed about the expected survival in the long term. Recommendations in treating patients with perforated diverticulitis with generalized peritonitis are mainly based on small or retrospective studies and short-term outcome. For many surgeons HP remains the standard treatment for this stage of disease, although a prospective multicenter evaluation of surgical procedures demonstrated that HP was associated with significantly more postoperative complications and a 1.8-fold increase in the likelihood of death compared with PA [17]. Fear of anastomotic leakage deters many surgeons from performing PA. As the operations were classified as emergency and hence may be undertaken outside office hours, 
not all procedures were performed by a specialist colorectal surgeon. It is known that noncolorectal surgeons prefer HP in the case of emergency surgery for colorectal surgery [18]. This preference is mainly based on the severity of disease and comorbidity and age of the patient in relation to the surgeon's experience in this field of surgery.

Patients who undergo HP have to deal with a colostomy. Additional stoma care might be a bridge too far for these (older) patients, as they face the physical (dehydration, leakage, parastomal hernia) and psychological (lifestyle alterations) challenges that are associated with having a stoma [19]. Probably they will never return to their prehospital health state owing to the stoma. Reversal of HP is associated with significant morbidity and even mortality $[19,20]$, especially in patients in a poor general condition with several comorbidities [7,21]. This is one of the reasons why HP often leaves the patient with a permanent colostomy.

It seems that PA for perforated diverticulitis compares equally well to HP in terms of longterm survival. Again it is essential to realise that high risk patients (higher ASA classification, older age, higher Hinchey score and MPI) were more likely to undergo HP. Nevertheless, after adjusting for these factors, survival after PA or HP was still not significantly different. Long-term survival was only related to patients' characteristics such as higher age and ASA classification III and IV. It was just this group of patients that was prominently present. Almost half of the patients was ASA III or higher and one third was older than 75 years. Within 6 years almost two third of these patients had died. One might postulate that particularly older patients with more comorbidities will present with a more severe inflammatory reaction causing perforation leading to death. This could also be an explanation for the statement that almost $80 \%$ of the patients present with perforated diverticulitis as the first manifestation of their diverticular disease [22]. The fist attack in older ASA III and IV patients is especially hazardous.

All these aspects including short- and long-term survival, stoma care and patients' characteristics must be taken into account before performing primary surgery. HP should no longer be considered as the most likely treatment for perforated diverticulitis in all patients. In the younger healthy patients, PA may be the procedure of choice for all stages of severity of perforated diverticulitis when all short- and long-term outcomes are considered. In addition, in patients of older age and with ASA classification III or higher, HP must seriously be considered the surgical procedure of choice, because the long-term survival is limited and restoration of bowel continuity is therefore not an issue. A study comparing the quality of life after both procedures is warranted.

In conclusion, long-term survival of patients after emergency surgery for acute perforated 
diverticulitis is limited and mainly caused by the poor general condition of these aged patients. The severity of the primary disease, the type of initial surgical procedure and the surgeon's experience do not influence long-term out-hospital survival.

\section{References}

1. Painter NS, Burkitt DP. Diverticular disease of the colon, a 20th century problem. Clin Gastroenterol $1975 ; 4: 3-22$

2. Mendeloff AI. Thoughts on the epidemiology of diverticular disease. Clin Gastroenterol 1986;15:85577

3. Jacobs DO. Clinical practice. Diverticulitis. N Engl J Med. 2007;357:2057-66

4. Makela J, Kiviniemi H, Laitinen S. Prevalence of perforated sigmoid diverticulitis is increasing. Dis Colon Rectum 2002;45:955-61

5. Chandra V, Nelson H, Larson DR, Harrington JR. Impact of primary resection on the outcome of patients with perforated diverticulitis. Arch Surg 2004; 139: 1221-3

6. Makela J, Kiviniemi H, Laitinen S. Prognostic factors of perforated sigmoid diverticulitis in the elderly. Dig Surg 2005;22:100-6

7. Salem L, Flum DR. Primary anastomosis or Hartmann's procedure for patients with diverticular peritonitis? A systematic Review. Dis Colon Rectum 2004;47:1953-64

8. Pisanu A, Cois A, Uccheddu A. Surgical treatment of perforated diverticular disease: evaluation of factors predicting prognosis in the elderly. Int Surg 2004;89:35-8

9. Zeitoun G, Laurent A, Rouffet F, Hay J, Fingerhut A, Paquet J, Peillon C, Research TF. Multicentre, randomized clinical trial of primary versus secondary sigmoid resection in generalized peritonitis complicating sigmoid diverticulitis. Br J Surg 2000;87:1366-74

10. Vermeulen J, Akkersdijk GP, Gosselink MP, Hop WC, Mannaerts GH, van der Harst E, Coene PP, Weidema WF, Lange JF. Outcome after emergency surgery for acute perforated diverticulitis in 200 cases. Dig Surg. 2007;24:361-6

11. Billing A, Frohlich D, Schildberg FW. Prediction of outcome using the Mannheim peritonitis index in 2003 patients. Br J Surg 1994;81:209-13

12. Hinchey EJ, Schaal PGH, Richards MB. Treatment of perforated diverticulitis of the colon. Adv Surg 1978;12:85-105

13. Ter Borg PC, Schalm SW, Hansen BE, van Buuren HR; Dutch PBC Study Group. Prognosis of ursodeoxycholic Acid-treated patients with primary biliary cirrhosis. Results of a 10-yr cohort study involving 297 patients. Am J Gastroenterol. 2006;101:2044-50

14. Morris CR, Harvey IM, Stebbings WSL, Hart AR. Incidence of perforated diverticulitis and risk factors for death in a UK population. Br J Surg. 2008;95:876-81

15. Vermeulen J, Gosselink MP, Hop WCJ, Lange JF, Coene PPLO, Harst E van der, Weidema WF, Mannaerst GHH. In-hospital mortality after emergency surgery for perforated diverticulitis (in Dutch). 
Ned Tijdschr Geneeskd 2009;153:1209-14

16. Richter S, Lindemann W, Kollmar O, Pistorius GA, Maurer CA, Schilling MK. One-stage sigmoid colon resection for perforated diverticulitis (Hinchey stages III and IV). World J Surg 2006;30:1027-32

17. Constantinides VA, Tekkis PP, Senapati A; Association of Coloproctology of Great Britain Ireland. Prospective multicentre evaluation of adverse outcomes following treatment for complicated diverticular disease. Br J Surg. 2006;93:1503-13

18. Zorcolo L, Covotta L, Carlomagno N, Bartolo DC. Towards lowering morbidity, mortality and stoma formation in emergency colorectal surgery: the role of specialization. Dis Colon Rectum 2003;46:14618

19. Gooszen AW, Geelkerken RH, Hermans J, Lagaay MB, Gooszen HG. Quality of life with a temporary stoma: ileostomy vs. colostomy._Dis Colon Rectum. 2000;43:650-5

20. Vermeulen J, Coene PPLO, Hout NM van, Harst E van der, Mannaerts GHH, Weidema WF, Lange JF. Restoration of bowel continuity after surgery for acute perforated diverticulitis: should Hartmann's procedure be considered a one-stage procedure? Colorectal Dis 2009;11:619-24

21. Banerjee S, Leather AJM, Rennie JA, Samano N, Gonzalez JG, Papagrigoriadis S. Feasibility and morbidity of reversal of Hartmann's. Colorectal Dis 2005;7:454-9

22. Hart A, Kennedy J, Stebbings W. How frequently do large bowel diverticula perforate? An incidence and cross-sectional study. Eur J Gastroenterol Hepatol 2000;12:661-6

\section{Tables}

Table 1. 
Characteristics of patients who survived emergency surgery for perforated diverticulitis $(n=250)$.

$$
\mathrm{n}(\%)
$$

\begin{tabular}{ll}
\hline Entire Cohort & 340 \\
Postoperative in-hospital death & $90(26)$ \\
Survivors from initial surgery & 250 \\
Diseased during follow up & $90 / 160(36 / 74)$ \\
(yes/no) & \\
\hline Gender (male/female) & $110 / 140(44 / 56)$ \\
Age (median (range)) & $66(23-95)$ years \\
\hline ASA & \\
I & $60(24)$ \\
II & $93(37)$ \\
III & $69(28)$ \\
IV & $28(11)$ \\
\hline MPI & \\
$<26$ & $224(90)$ \\
=26 & $26(10)$ \\
\hline Hinchey score & \\
I & $58(23)$ \\
II & $56(22)$ \\
III & $108(43)$ \\
IV & $28(11)$ \\
\hline Type of surgery &
\end{tabular}

\begin{tabular}{ll} 
Type of surgery & $163(65)$ \\
HP & $80(32)$ \\
PA & $7(3)$ \\
Other & $17(5-137)$ days \\
\hline Hospital stay, median (range) & $1(0-61)$ days \\
ICU stay, median (range) &
\end{tabular}

Values in parentheses are percentages of patients unless indicated otherwise.

ASA $=$ American Society of Anaesthesiologist classification; MPI = Mannheim peritonitis index; HP = Hartmann's procedure; $\mathrm{PA}=$ resection with primary anastomosis; $\mathrm{ICU}=$ Intensive care unit 
Table 2.

Causes of death in 90 patients having survived emergency surgery for perforated diverticulitis 
and discharged from hospital $(\mathrm{n}=86)$.

\begin{tabular}{ll} 
Cause of death & $\mathrm{n}(\%)$ \\
& \\
\hline & \\
Myocardial Infarction / Cardiac decompensation & $17(19)$ \\
Age and general condition & $15(17)$ \\
Cancer & $14(16)$ \\
Exacerbation COPD / Pneumonia & $8(9)$ \\
Cerebro-vascular accident & $5(6)$ \\
Complicated reversal of Hartmann's procedure (HP) & $5(6)$ \\
Recurrent complicated diverticulitis & $3(3)$ \\
Urosepsis / Multi organ failure & $3(3)$ \\
Complicated surgery for ileus & $2(3)$ \\
Pancreas / Biliary tract infection & $2(3)$ \\
Gastric ulcer perforation & $1(1)$ \\
Ruptured abdominal aorta & $1(1)$ \\
Unknown & $14(16)$ \\
\hline
\end{tabular}

Values in parentheses are percentages.

Table 3.

Multivariate Cox's regression analysis for long-term mortality after surviving initial 
emergency surgery for perforated diverticulitis.

\section{Long-term mortality}

HR $95 \% \mathrm{CI} \quad \mathrm{p}$

\begin{tabular}{|c|c|c|c|}
\hline \multicolumn{4}{|c|}{ Surgical procedure } \\
\hline HP & 1 & & \\
\hline PA & 0.54 & $0.3-1.04$ & 0.07 \\
\hline \multicolumn{4}{|l|}{ Gender } \\
\hline Male & 1 & & \\
\hline Female & 1.1 & $0.6-1.8$ & 0.87 \\
\hline \multicolumn{4}{|l|}{ Age (years) } \\
\hline$<53$ & 1 & & $<0.001$ \\
\hline 53-66 years & 1.1 & $0.4-2.5$ & 0.99 \\
\hline $67-75$ years & 2.7 & $1.1-5.7$ & 0.05 \\
\hline$>75$ years & 4.1 & $1.6-8.4$ & 0.002 \\
\hline \multicolumn{4}{|c|}{ ASA (vs. ASA I) } \\
\hline I & 1 & & 0.002 \\
\hline II & 1.8 & $0.7-3.9$ & 0.29 \\
\hline III & 4.2 & $1.5-8.6$ & 0.004 \\
\hline IV & 4.8 & $1.5-11.2$ & 0.006 \\
\hline \multicolumn{4}{|c|}{ Hinchey score } \\
\hline I & 1 & & 0.75 \\
\hline II & 1.2 & $0.5-2.5$ & 0.71 \\
\hline III & 1.1 & $0.6-2.2$ & 0.79 \\
\hline IV & 0.54 & $0.2-1.9$ & 0.45 \\
\hline
\end{tabular}

Data given are Hazard ratios (HR) with $95 \%$ confidence intervals $(95 \% \mathrm{CI})$.

$\mathrm{HP}=$ Hartmann's procedure; $\mathrm{PA}=$ resection with primary anastomosis; ASA = American

Society of Anaesthesiologist classification

\section{Figures}




\section{Figure 1.}

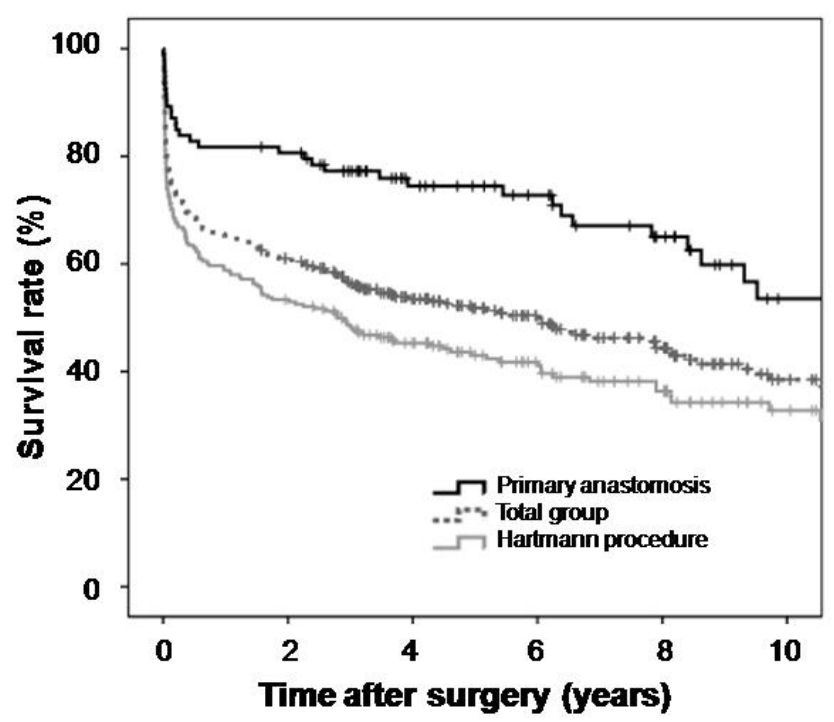

Kaplan-Meier plot illustrating survival of all 340 patients who presented with acute perforated diverticulitis and underwent emergency surgery between 1990 and 2005. Patients who underwent Hartmann's procedure $(\mathrm{n}=238)$ and those who underwent primary anastomosis $(n=93)$ are also shown separately.

\section{Figure 2.}

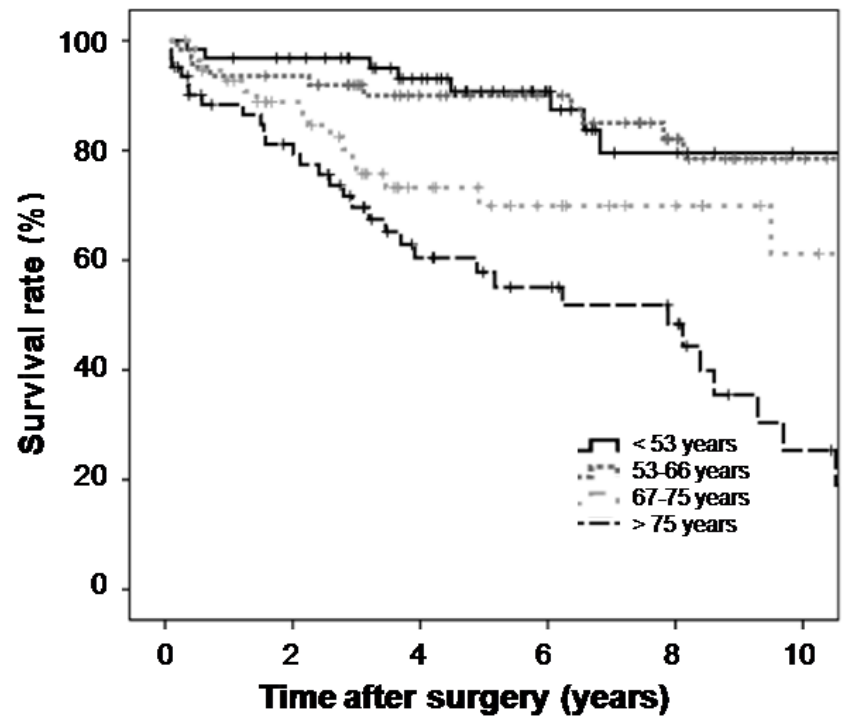

Kaplan-Meier plot illustrating survival of patients $(n=250)$ after successful emergency surgery for perforated diverticulitis according to age (log-rank test, $\mathrm{p}<0.001)$. 


\section{Figure 3.}

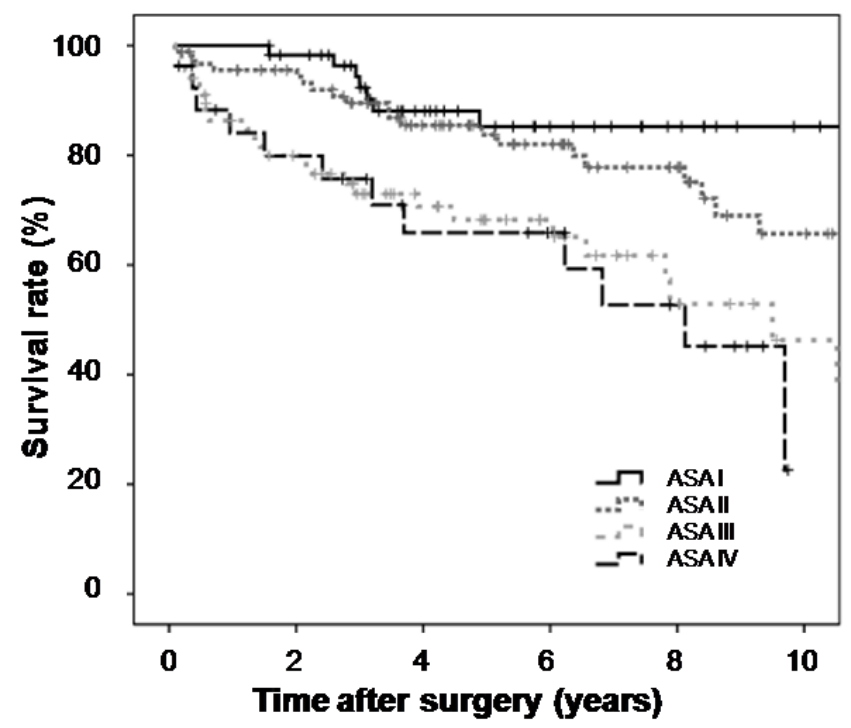

Kaplan-Meier plot illustrating survival of patients $(n=250)$ after successful emergency surgery for perforated diverticulitis according to ASA classification (overall p-value <0.001).

\section{Figure 4.}

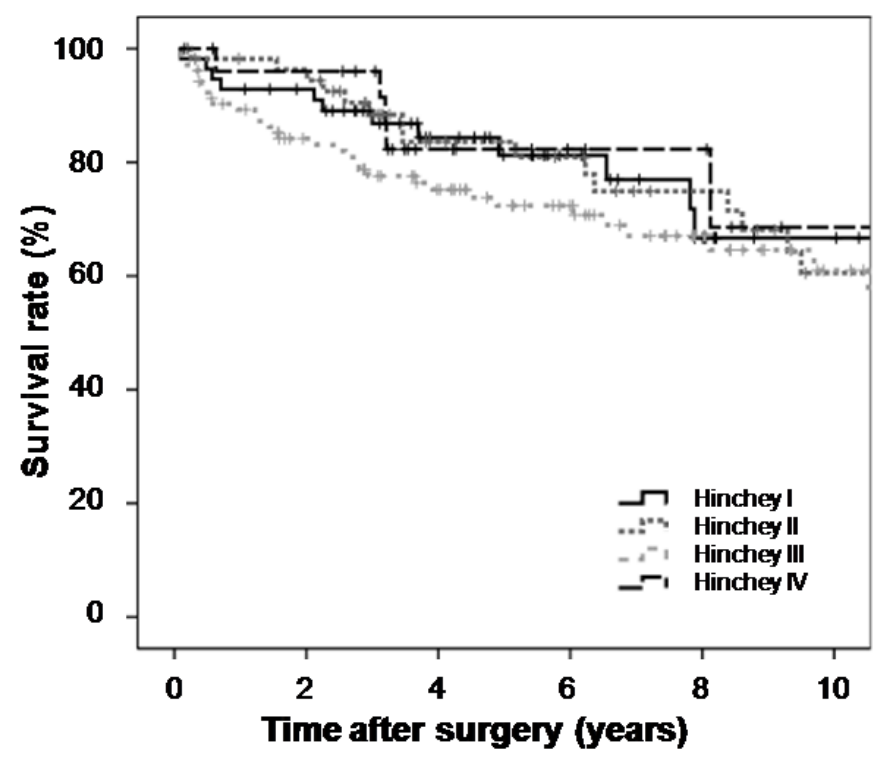

Kaplan-Meier plot illustrating survival of patients $(n=250)$ after successful emergency surgery for perforated diverticulitis according to Hinchey score $(p=0.27)$. 


\section{Figure 5.}

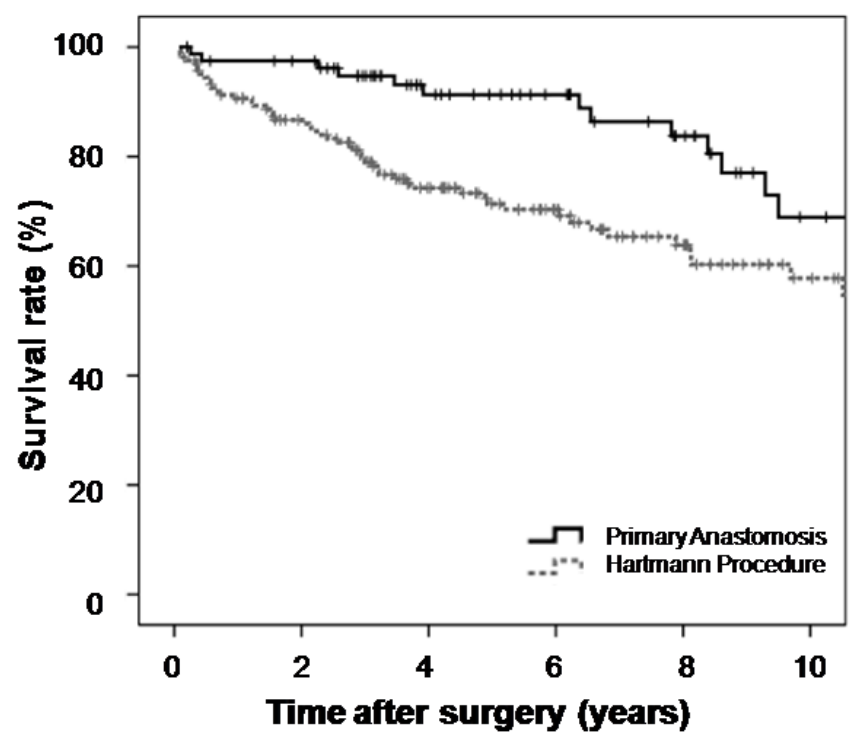

Kaplan-Meier plot illustrating long-term survival of patients after successful emergency surgery for perforated diverticulitis according to type of surgery (log-rank test, $\mathrm{p}=0.005$ ). Hartmann's procedure, $n=163$; Primary anastomosis, $n=80$.

\section{Figure 6.}

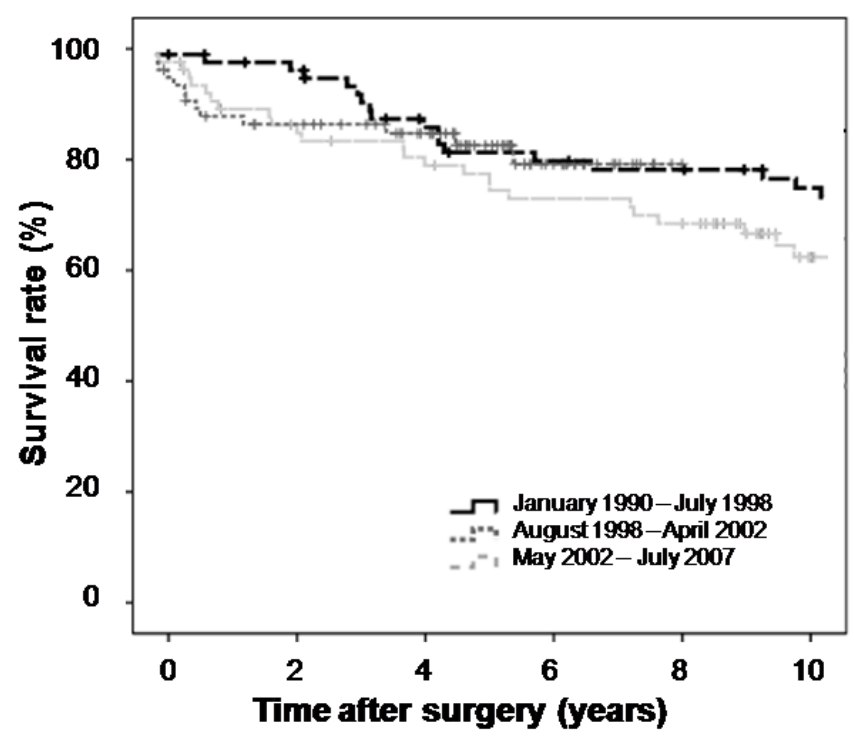

Kaplan-Meier plot illustrating survival of patients $(n=250)$ after successful emergency surgery for perforated diverticulitis according to the period in which the operation had taken place (log-rank test, $\mathrm{p}=0.27)$. 


\section{Figure 7.}

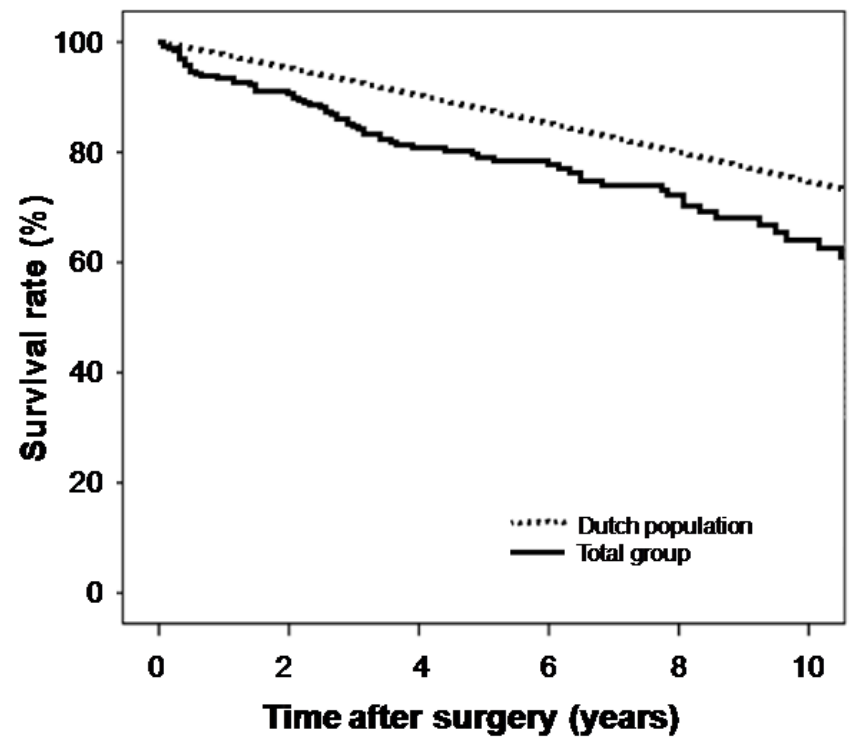

Kaplan-Meier plot illustrating survival of patients $(n=250)$ after successful emergency surgery for perforated diverticulitis and the expected matched sex-, age- and calendar time-specific survival of the general Dutch population (log-rank test, $\mathrm{p}=0.005$ ).

\section{Figure 8.}

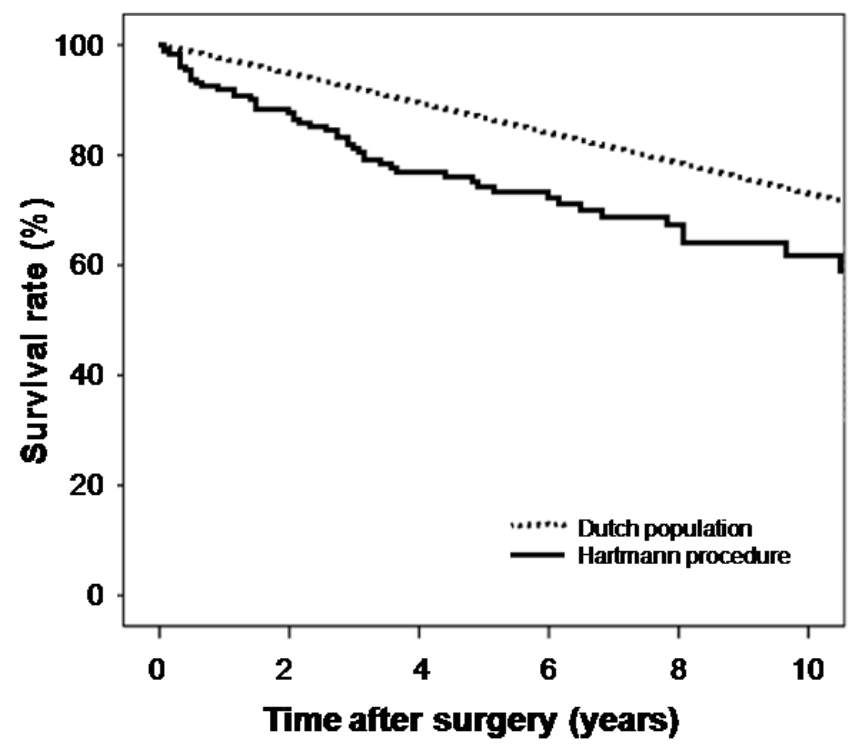

Kaplan-Meier plot illustrating survival of patients $(n=163)$ after Hartmann's procedure for perforated diverticulitis and the expected matched sex-, age- and calendar time-specific survival of the general Dutch population $(n=163)$ (log-rank test, $p=0.02)$. 
Figure 9.

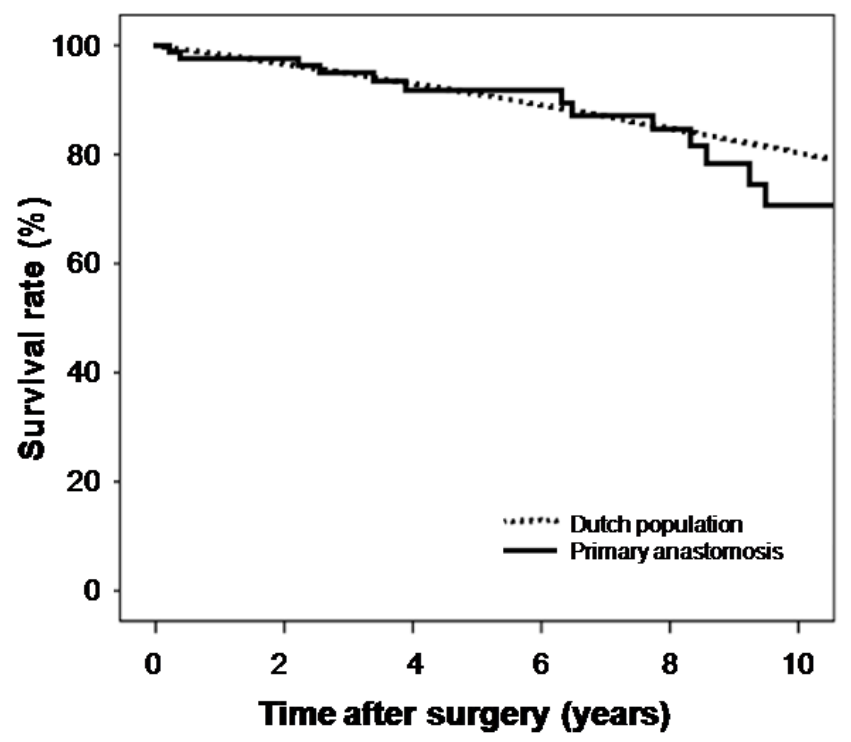

Kaplan-Meier plot illustrating survival of patients $(n=80)$ after resection with primary anastomosis for perforated diverticulitis and the expected matched sex-, age- and calendar time-specific survival of the general Dutch population $(n=80)$ (log-rank test, $\mathrm{p}=0.852)$. 